\title{
De hombres y machos: el género y las masculinidades en la vida cotidiana
}

\author{
Ricardo Ayllón González
}

\section{Resumen}

En el presente artículo se definen los conceptos de género, masculinidades, masculinidad hegemónica, machismo y códigos culturales. Asimismo, se aborda el tema de las masculinidades alternativas y se habla acerca de la posibilidad del cambio en los hombres.

Palabras clave: género, masculinidad, machismo, masculinidad hegemónica.

\section{About men and machos: Gender and masCUlinities IN EVERYDAY LIFE}

\begin{abstract}
In this article the concepts of gender, masculinities, hegemonic masculinity, machismo and cultural codes are defined. In addition, the topic of alternative masculinities is addressed and the further possibility of change in men is discussed.
\end{abstract}

Keywords: gender, masculinity, machismo, hegemonic masculinity. 


\section{Ricardo Ayllón González}

Licenciado en Psicología por la Universidad Nacional Autónoma de México (UnAm) y Maestro en Educación Humanista por el Instituto Humanista de Psicoterapia Gestalt. Es especialista en Orientación Humanista y en Psicoterapia Gestalt por el Instituto Humanista de Psicoterapia Gestalt; en coordinación de grupos comunitarios desde la Terapia de Reencuentro, impartida por la Dra. Fina Sanz del Instituto de Terapia de Reencuentro de Valencia, España; y en la conducción de grupos de Hombres contra la Violencia Intrafamiliar desde el modelo CECEVIM, impartido por el Dr. Antonio Ramírez del Centro de Capacitación para Erradicar la Violencia Intrafamiliar Masculina, de San Francisco, California. Autor y coautor de diversos textos y guías para el trabajo con hombres elaborados en GENDES A.c. Ponente y tallerista en diversos eventos a nivel nacional e internacional. Es cofundador de GENDES A.c. y coordinador del programa de metodología en dicha institución. 


\section{Introducción}

Los días 8 y 9 de marzo de 2020 se llevó a cabo un hecho histórico: las mujeres de México se unieron para protestar en contra de la violencia que se vive hacia ellas, y en rechazo a la impunidad y al silencio cómplices. La consigna "El 9, ni una se mueve" fue real: las calles de la Ciudad de México extrañaron a las mujeres, porque su ausencia fue evidente. Como una respuesta obligada y necesaria, diversas instituciones académicas, de gobierno, privadas y sociales convocaron a algunos hombres para iniciar una primera reflexión o, en algunos casos, continuarla, acerca de las masculinidades, el machismo, la responsabilidad que

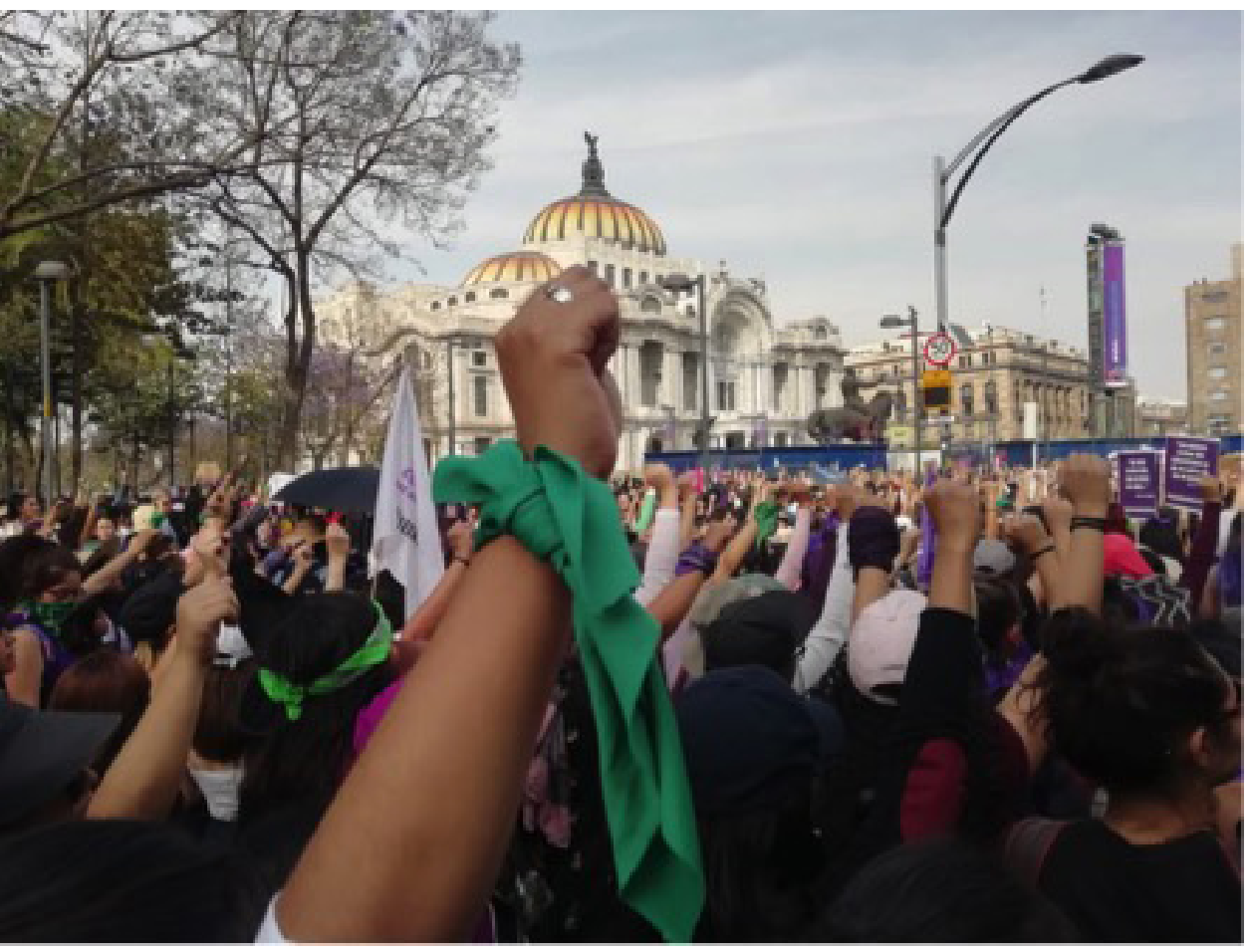
nos compete ante la realidad social de la violencia -a propósito de la avasallante manifestación de mujeres-y lo que hemos hecho para frenarla.

Ocurrió de la misma manera que en los movimientos de mujeres de los años sesenta del siglo pasado: las reflexiones de los hombres van apareciendo; algunos van sumándose, dialogando y "cuestionándose" su ser hombres, a partir de que ellas levantan lavoz, buscan visibilizar las condiciones de explotación, segregación, subordinación, discriminación, desigualdad, opresión, exclusión y violencia que han experimentado a lo largo de la historia (Núñez, 2016). Este texto continuará contribuyendo con algunas de esas reflexiones.

\section{Del género a la masculinidad}

Es necesario revisar cómo nos hemos construido como hombres y la forma en que nos relacionamos tanto con las mujeres como con otros hombres; esto lo podemos abordar desde el concepto género.

Por género entendemos al sistema de significados, normas, prácticas y representaciones construidas social y culturalmente con base en características relacionadas con el sexo de las personas. Este conjunto de prácticas determina una serie de comportamientos asociados con tales características que derivan en atribuciones sociales impuestas a uno y otro sexo, involucrando relaciones de poder y desigualdad entre lo femenino y lo masculino (Leñero, 2010). 
Aprendemos a ser mujeres y hombres acorde al espacio físico, social y cultural en el que nos desarrollamos y convivimos. Es un proceso de aprendizaje complejo y dinámico que se refuerza y cambia constantemente a lo largo del curso de vida. Sin embargo, no sólo aprendemos, también enseñamos a otras y a otros cómo ser mujeres y hombres.

Por su parte, la masculinidad es el conjunto de todos los significados, las conductas y los códigos que se construyen socialmente y que se atribuyen a lo que un hombre "debe ser" (Connell, 2003). Son ideas, creencias que nos impone la sociedad y la cultura a los hombres en nuestras formas de ser, decir, actuar y pensar.

Sehabla de masculinidades(ver video 1), en plural, porquehay muchasformas de vivirse como hombres (Connell, 2003). Sin embargo, hay comportamientos que son comunes en gran parte de nosotros (De Keijzer, 2010):

- La actitud de sentirse superiores a las mujeres.

- Ser el principal proveedor económico de la familia.

- El uso de alguna forma de violencia para imponerse y ejercer un control hacia otras y otros.

- Creer que la heterosexualidad es vista como la única forma de vivir la sexualidad, además de poner al sexo y, en especial, al coito como centro y/o eje de las relaciones con las mujeres (Heilman, Barker y Harrison, 2017).

Video 1. Masculinidades.

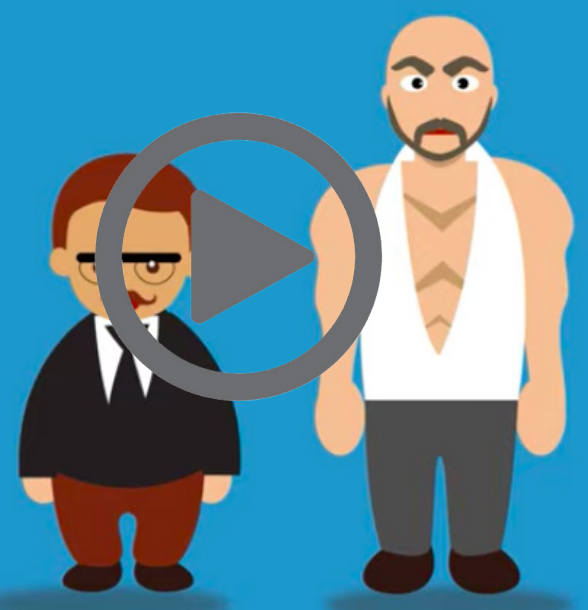

Un tipo de masculinidad es la masculinidad hegemónica, la cual se refiere a los modos de ser hombre que se valoran socialmente y actúan como punto de referencia para los hombres, a la vez que buscan y hacen legítima la subordinación de las mujeres respecto de los hombres, haciendo de la violencia un eje rector para mantener el control y ejercer un abuso de poder sobre todo lo referente al mundo femenino (Connell, 2003). 
Por otra parte, existen las Ilamadas masculinidades subordinadas, en las que se incluirían a quienes se considera inferiores como los niños, adultos mayores, personas de la comunidad LGBTTRQ, ${ }^{1}$ entendiendo que éstos son subordinados a aquéllos que ostentan la masculinidad hegemónica (Connell, 2003).

Esta serie de conductas que tienen como fin dominar a las mujeres o a esos grupos subordinados se le llama machismo. Éste se compone de conductas, comportamientos y creencias que promueven, reproducen y refuerzan diversas formas discriminatorias contra las mujeres. Se construye a través de la polarización de los roles y estereotipos que definen lo masculino de lo femenino. Su principal característica es la degradación de lo femenino; su mayor forma de expresión es la violencia en cualquiera de sus tipos en contra de las mujeres (Ramírez, 2000).

En México se ejerce más violencia contra las mujeres que en otros. De hecho, en las calles de nuestro país, las mujeres no pueden caminar seguras, ya que hay un "permiso" social que repercute en todos los aspectos, desde que sean invadidas con palabras o con "piropos", hasta el feminicidio.

Las ideas acerca de cómo debemos ser los hombres también nos coloca en una triada de riesgo hacia mujeres y niños, hacia otros hombres y hacia uno mismo (OPS, 2019). Nos pone en situaciones de riesgo y, paradójicamente, nos hace más vulnerables (ver video 2). Según datos de la ops (2017), los hombres tienden a morir en mayor proporción (718.8/100 000 hombres frente a 615.1/100 000 mujeres). Sin embargo, tres de las causas principales de muerte están vinculadas al ejercicio de la masculinidad, y sus consecuencias muestran una enorme diferencia: la violencia interpersonal (en la que destacan los homicidios, con una razón de 7 hombres por mujer), los traumatismos debidos al tránsito (3 por 1) y la cirrosis hepática (más del doble), cuya primera causa directa es el consumo de alcohol. Estos motivos se asocian a las conductas esperadas en los hombres en virtud de una masculinidad hegemónica con expresiones tóxicas (ops, 2017, p. 7).

Video 2. Por qué los derechos de las mujeres son importantes para los hombres.
1'LGBTTQI son las siglas alusivas a las comunidades de Lesbiana, Gay, Bisexual, Transexual, Transgénero, Travesti, Queer e Intersexual (Redacción de Animal Político, 2019).

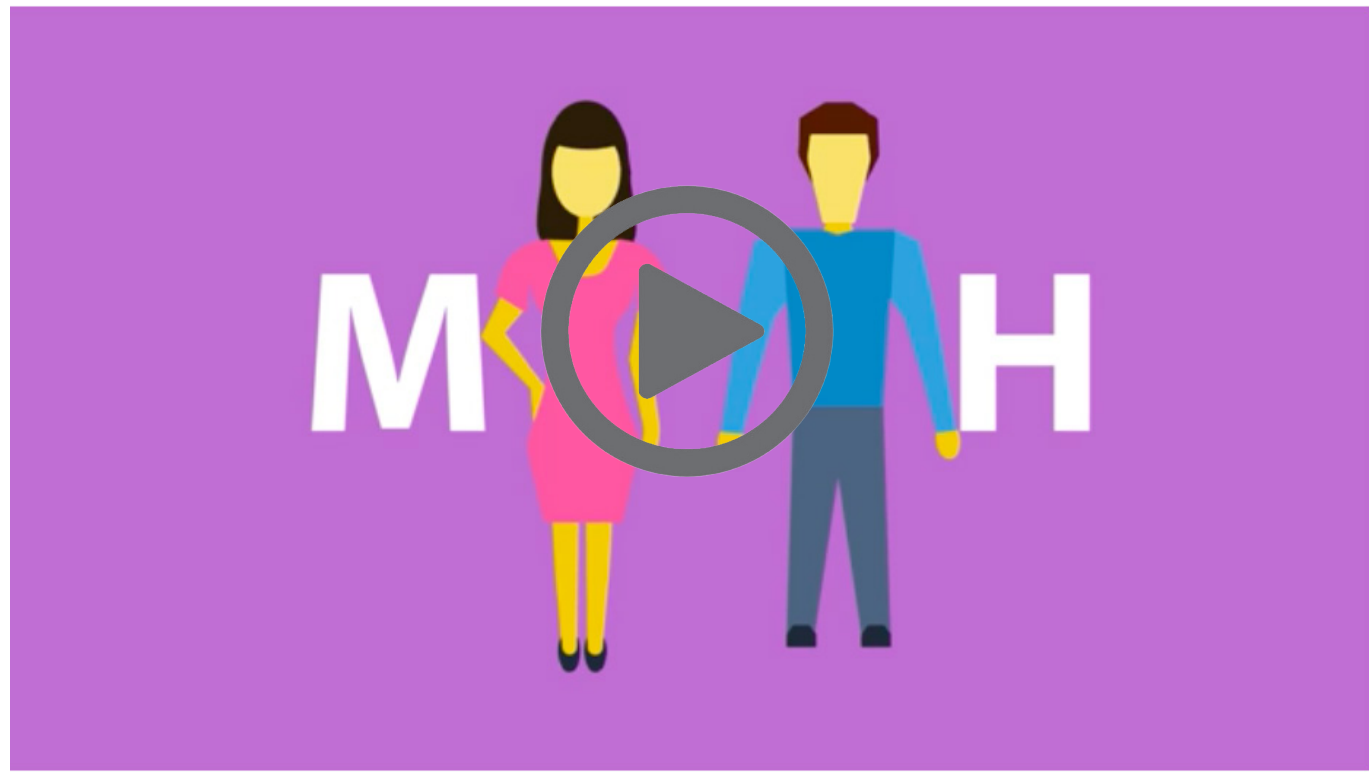




\section{Códigos culturales}

A las ideas o creencias que guían y dictan el comportamiento de los hombres se les llama códigos (Ramírez, 2000). Existen códigos que forman parte de la vida social y de la cultura que vamos aprendiendo y reproduciendo en la vida cotidiana, en nosotros mismos y en nuestros vínculos. Algunos de estos códigos culturales para los hombres son:

- Tener fortaleza física.

- Expresar emociones como el enojo, la ira, la frustración y, por otro lado, limitar la expresión de la tristeza, el afecto, el miedo, la ternura.

- El uso de la razón sobre las emociones.

- Sentirse invulnerables.

- Poco o nulo cuidado del cuerpo, la salud física y emocional.

- Someterse a situaciones de alto riesgo.

- Estar en espacios públicos (en la calle y no dentro de casa).

- No hacerse cargo de actividades domésticas y cuidado de otros.

- La falta de empatía por las emociones de otras personas.

- Mirar a las mujeres como un objeto sexual.

- Uso del albur como un recurso para someter y denigrar a otros hombres.

- Uso del alcohol y otras drogas (De Keijzer, 2010; Heilman, Barker y Harrison, 2017).

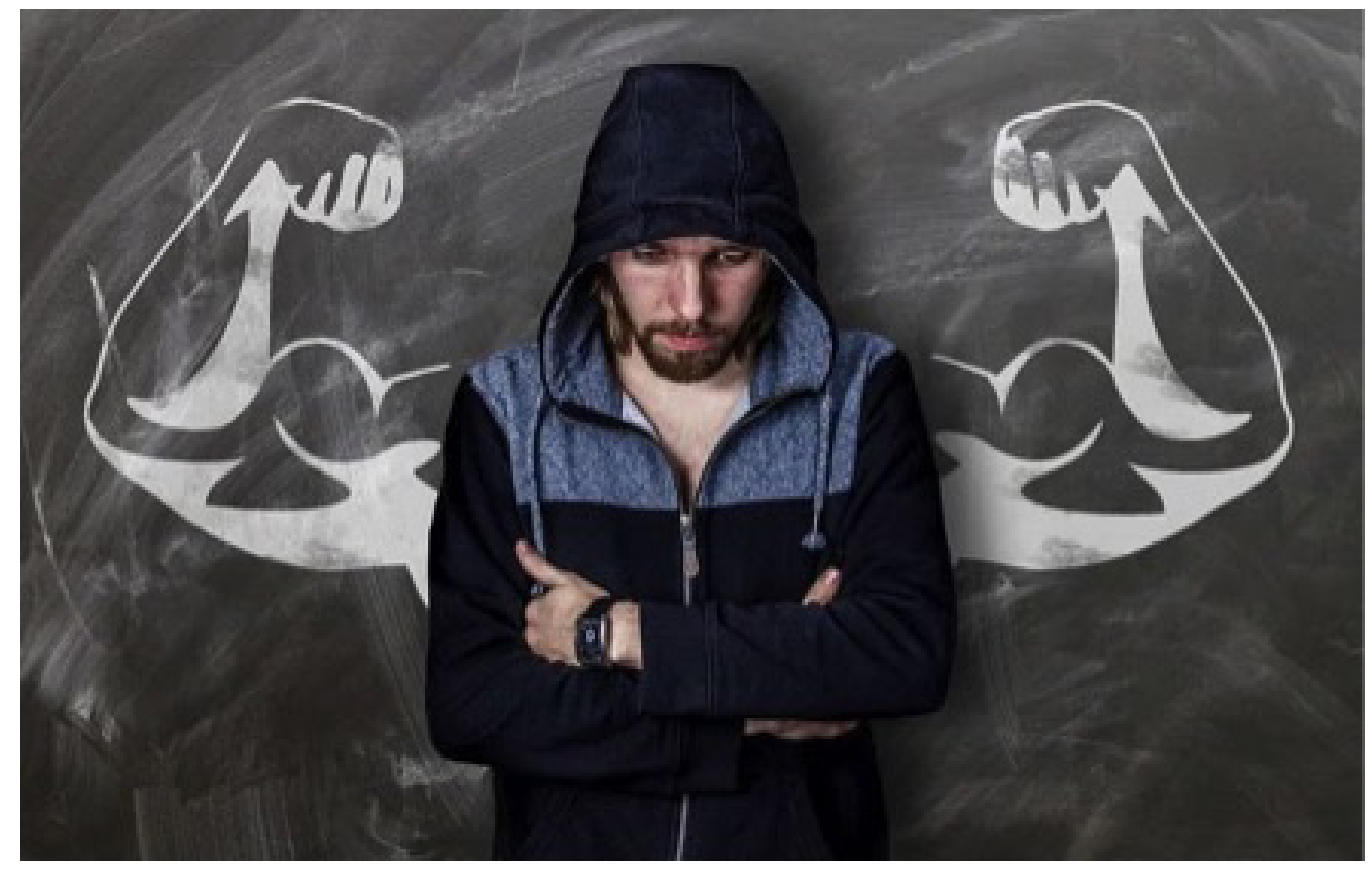


Las creencias acerca del comportamiento de los hombres son producto de aprendizajes heredados y reproducidos casi incuestionablemente generación tras generación. No obstante, es importante saber que, en cuestión de aprendizajes, podemos también desaprenderlos y aprender otras formas de vivirnos como hombres.

\section{Las posturas de los hombres ante el tema de género y masculinidades}

En nuestro país podemos ver en los hombres distintas formas de reaccionar ante las reflexiones y los temas antes mencionados:

- El hombre fiel al machismo. ${ }^{2}$ Es el hombre que, ya sea que desconozca toda información al respecto del género y las masculinidades y siga su vida de acuerdo a los códigos hegemónicos, o bien, que conozca del tema y lo viva con enojo y le provoque un verdadero problema los posibles cambios en las formas de relacionarse.

- El macho "progre". Es el hombre que tiene un discurso de género e igualdad, pero sus actitudes siguen basándose en creencias y prácticas machistas (Canales, L. y Redacción Malvestida, 2018).

- Hombre en proceso de cambio. ${ }^{3}$ Es el hombre que sigue ejerciendo violencia en algunas de sus formas, aunque promueve una reflexión interna y trata de asumir una responsabilidad para realizar acciones de transformación en su persona y en sus vínculos desde una masculinidad alternativa.

\section{Masculinidad alternativa}

En los últimos años, se ha propuesto el término de masculinidad alternativa ${ }^{4}$ al modelo hegemónico o machista de ser hombre.

La masculinidad alternativa es una corriente/discurso que promueve conductas y actitudes entre los varones orientadas hacia la igualdad entre hombres y mujeres en todos los ámbitos de las relaciones interpersonales. Implica los siguientes aspectos:

- Cuestionar el modelo hegemónico de ser hombre.

${ }^{2}$ Término propuesto por el autor.

${ }^{3}$ Término propuesto por el autor.

${ }^{4}$ Término propuesto por GENDES A.C.
- Detener la violencia masculina.

- Aprender a solucionar pacíficamente los conflictos.

- Promover el autocuidado y cuidado de los otros y otras.

- Contactar con las emociones.

- Ejercer una vida sexual sana, responsable y global, más que sólo genital. 
La masculinidad alternativa representa una posibilidad de cuestionar y modificar las realidades del ser hombre.

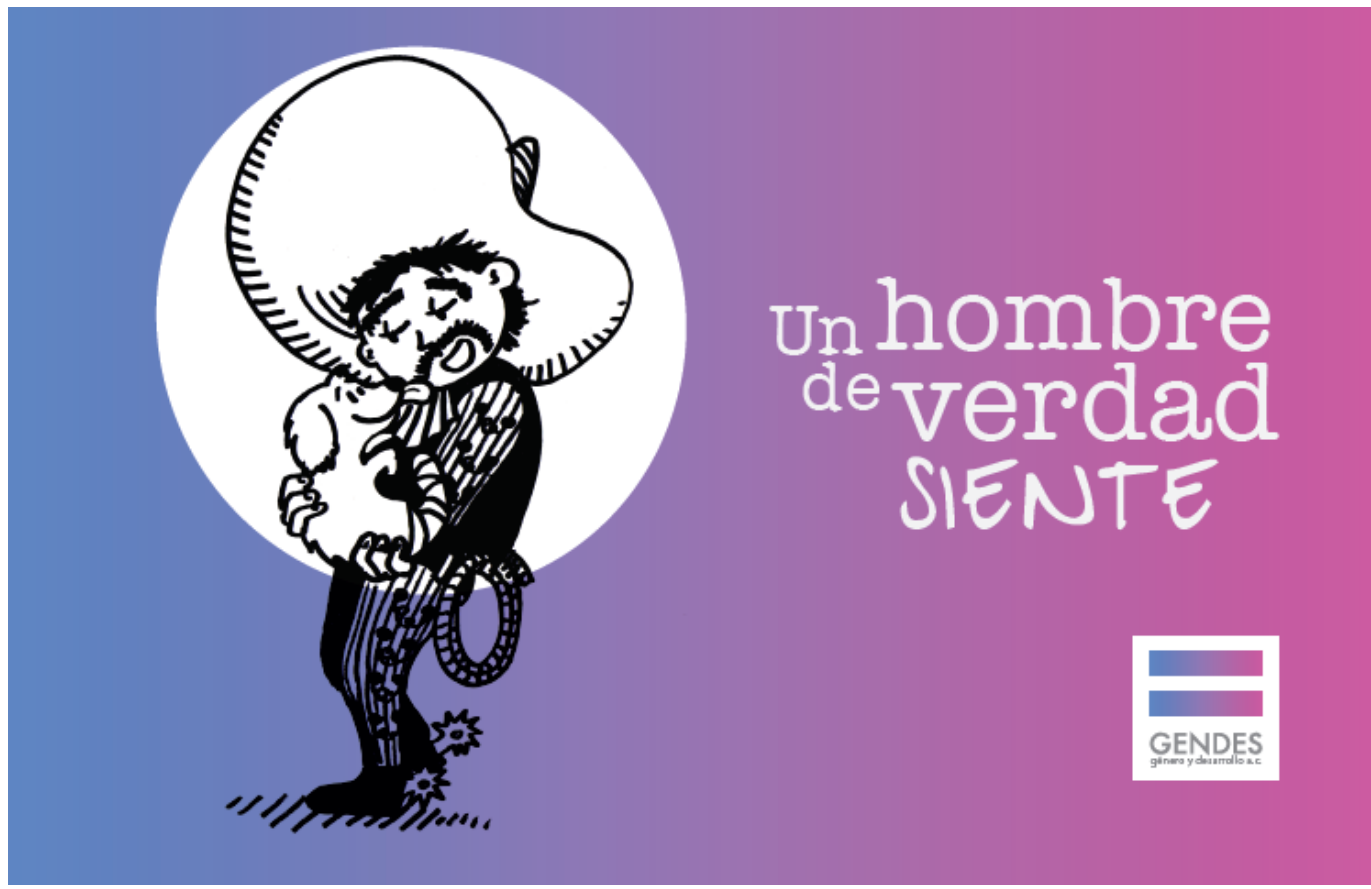

\section{El cambio es posible}

En mi práctica como psicólogo y psicoterapeuta considero que la posibilidad de cambio es posible, en especial a partir de situaciones de crisis. Algunas de estas crisis pueden ser la separación, divorcio o ruptura de la pareja, el mandato judicial de restricción a la familia, alguna situación de enfermedad o muerte, el nacimiento de algún hijo o hija, situaciones de desempleo o algunas crisis de edad, ya sea que se llegue a los treinta, cuarenta o cincuenta años.

En este período de contingencia sanitaria también han aflorado e incluso exacerbado las situaciones críticas, debido a que las condiciones en las que se pueden seguir los mandatos sociales de ser hombre se han modificado. Los hombres se encuentran en casa confinados y no en las calles, o bien, en el espacio público. En algunos casos se ha trastocado su papel de proveedor, y no saben cómo manejar en su convivencia cotidiana la frustración, estrés, miedo e incertidumbre que la amenaza a la salud y a la vida les genera.

Por lo tanto, también la contingencia sanitaria puede ser vista como un

${ }^{5}$ Se puede consultar la continuación del video Los hombres y el \#AislamientoSinViolencia: Estar bien con la familia en: parte 2 y parte 3 . momento de oportunidad y apertura; un momento coyuntural para promover acciones de cambio en los hombres. Como hemos venido haciendo, en esta época se han utilizado campañas, videos (ver video 3), ${ }^{5}$ carteles con mensajes para acercar a los hombres a algún tipo de atención virtual y telefónica, promoviendo además la asistencia presencial posterior a la emergencia de salud que vivimos. 
Video 3. Los hombres y el \#AislamientoSinViolencia: Estar bien con la familia (parte 1).

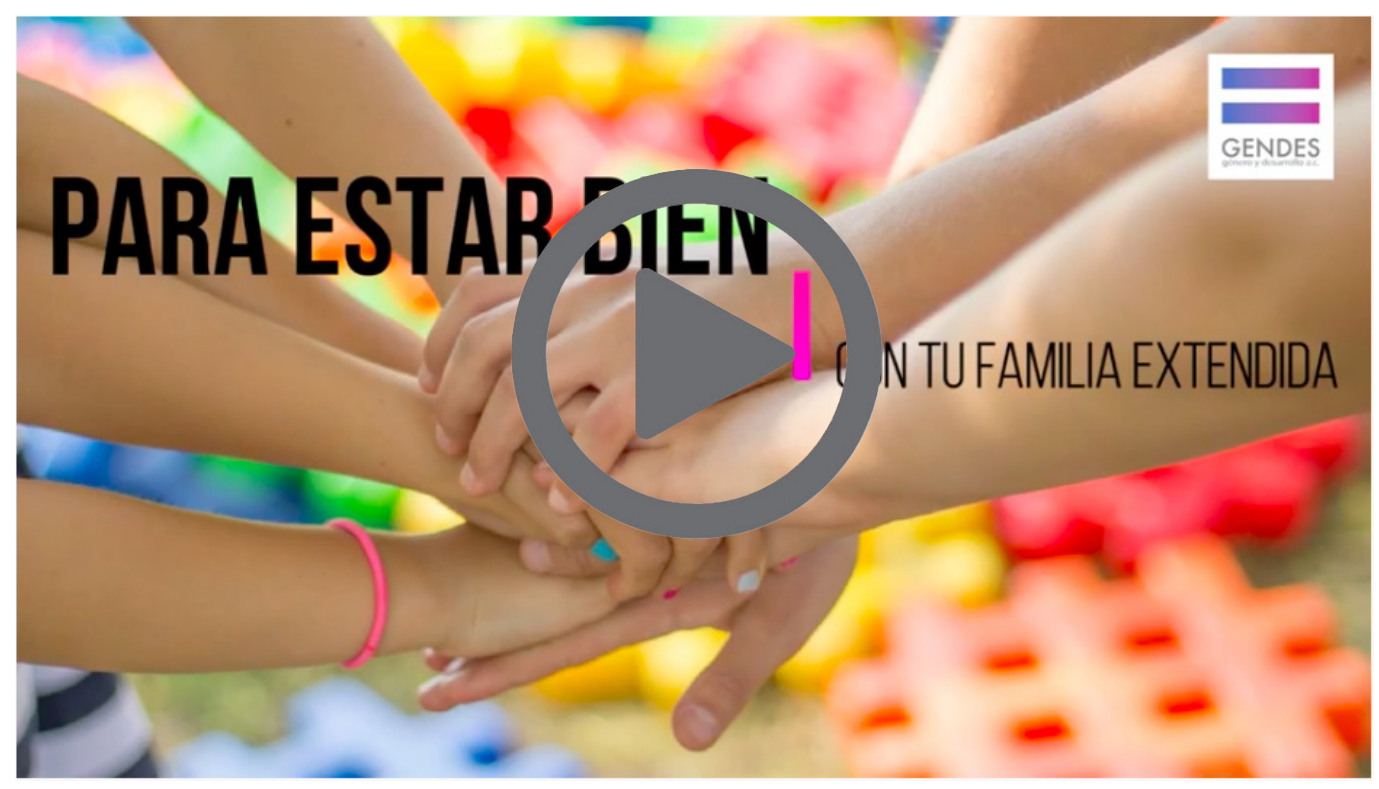

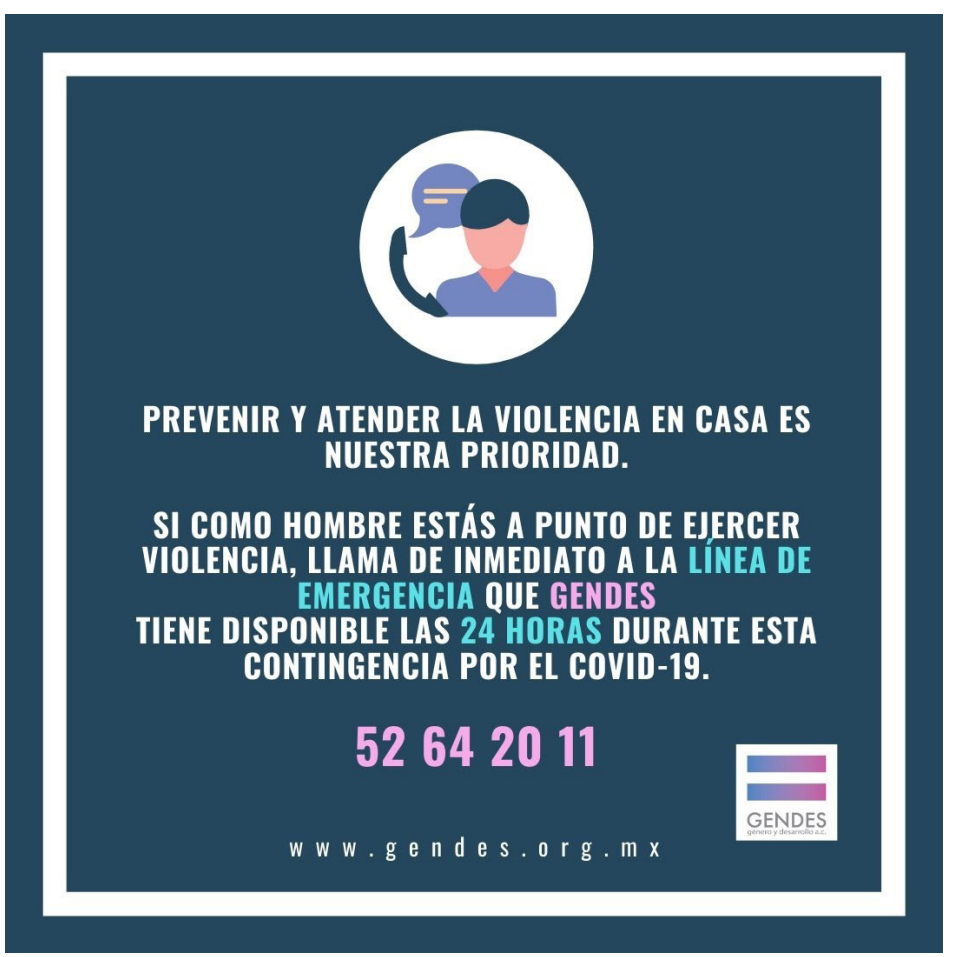

${ }^{6}$ Buen trato. Término propuesto por Fina Sanz (2016), que se define como "una forma de expresión del respeto y amor que merecemos y que podemos manifestar en nuestro entorno, como un deseo de vivir en paz y desarrollarnos en salud, bienestar y goce" (p. 47).

\section{Amaneradecierreyrecomendaciones}

Consideramos fundamental que los hombres de todas las edades iniciemos o continuemos con la reflexión y el trabajo personal y de transformación que empezamos a ver a inicios del mes de marzo. Ahí encontramos a muchos hombres preguntarse: ¿qué puedo hacer yo?, ¿qué me toca hacer como hombre? Un primer paso es detenerte a pensar de dónde provienen tus ideas, tus creencias sobre ser hombre y sobre ser mujer, así como cuestionar si son formas hegemónicas o si van orientadas hacia la igualdad y la no violencia.

Buscar información tanto teórica como de trabajo vivencial para promover el cambio puede ser el siguiente paso, ya sea buscando apoyo de alguna persona especializada o asistiendo a alguna de las instituciones sociales que brinden esta ayuda. Así podrás identificar si estás cometiendo alguna forma de violencia, y tener presente que ésta va desde la física hasta la psicológica.

El cambio es posible: otra forma de ser hombre, desde el respeto y el buen trato ${ }^{6}$ lo puedes iniciar en ti y en tus vínculos, aún con el contexto en el que vivimos. Recuerda que con tu propio comportamiento también enseñas a otras personas cómo ser hombres y cómo relacionarse con los demás. Desde una 
cultura diferente, de buen trato, se podrán generar actitudes de responsabilidad activa hacia el cuidado, el respeto, la noviolencia ${ }^{7}$ y la igualdad, para construir en conjunto una sociedad mejor.

\section{Referencias}

* Canales, L. y Redacción Malvestida. (2018, 11 de abril). Diccionario feminista para principiantes. Malvestida. https://malvestida.com/2018/04/diccionario-feministaprincipiantes/

- Connell, R.W. (2003). Masculinidades. Universidad Nacional Autónoma de México (UNAM).

* Cascón, P. (s.f.). Acción directa noviolenta y Desobediencia civil. Illacrua, 92, Noviolència. http://www.noviolencia.org/publicaciones/illacrua.pdf

* De Keijzer, B. (2010). Masculinidades, violencia, resistencia y cambio [tesis doctoral en Salud Mental Comunitaria (mimeo), Instituto de Investigaciones Psicológicas, Universidad, Veracruzana: Xalapa, México].

* Heilman, B., Barker, G. y Harrison, A. (2017). La caja de la masculinidad: un estudio sobre lo que significa ser hombre joven en Estados Unidos, el Reino Unido y México: hallazgos clave. Promundo-u.s. y Unilever.

* Leñero, M. (2010). Tercera llamada: orientaciones de género para la vida cotidiana. unAm, Programa Universitario de Estudios de Género. Fondo de Desarrollo de las Naciones Unidas para la Mujer.

* Núñez, G. (2016). Los estudios de género de los hombres y las masculinidades: ¿qué son y qué estudian?. Culturales, 4(1), 9-31. http://www.scielo.org.mx/scielo. php?script=sci arttext\&pid=S1870-11912016000100009\&lng=es\&tlng=es

fusiona las dos palabras y evita la separación en dos con un espacio o un guión, que sólo supondría el énfasis en la negación de la violencia directa; en cambio, este término constituye un proyecto en positivo de transformación radical de la sociedad y de nosotros y nosotras mismas. Noviolencia equivaldría a la traducción del término hindú ahimsa. En una sola palabra es

como vienen usándola desde hace décadas los diferentes movimientos antimilitaristas y noviolentos para referirse a una definición en positivo que la presenta como: una forma de vida, una estrategia de lucha

política, una herramienta para

enfrentar los conflictos y un modelo de sociedad. Tomado de Cascón, P.

\section{Cómo citar este artículo}

* Ayllón González, Ricardo. (2020, julio-agosto). De hombres y machos: el género y las masculinidades en la vida cotidiana. Revista Digital Universitaria (RDU), 21(4). DOI: http://doi.org/10.22201/codeic.16076079e.2020.21.4.5

Recepción: 27/04/2020. Aceptación: 22/05/2020. 